\title{
Los fondos de conocimiento en el desarrollo de la interculturalidad. Dilemas y paradojas ante los efectos de la covid-19 en alumnado inmigrante en situación de vulnerabilidad
}

\author{
Funds of knowledge in the development of interculturality. Dilemmas and \\ paradoxes in the face of the effects of covid-19 on immigrant students in vulnerable \\ situations
}

\author{
María José Alcalá del Olmo Fernández ${ }^{1}$ \\ Juan José Leiva Olivencia ${ }^{2}$
}

\begin{abstract}
Resumen
En la actualidad, los sistemas educativos mundiales atraviesan momentos de incertidumbre originados por la irrupción de una crisis socio-sanitaria motivada por la COVID-19, lo que ha llevado a desplegar todo un conjunto de soluciones de educación en una modalidad híbrida con la que asegurar la continuidad pedagógica en las mejores condiciones posibles. Esta situación sin precedentes ha generado un gran impacto en todos los agentes educativos, afectando de forma significativa al alumnado más vulnerable. El propósito de este trabajo es analizar los efectos de la pandemia motivada por la COVID-19 en el alumnado inmigrante en situación de vulnerabilidad, reflexionando en torno a los Fondos de Conocimiento como instrumentos esenciales para visibilizar a las minorías étnicas y tratando de indagar, de una forma crítica y reflexiva, en aquellas acciones que pueden resultar determinantes para garantizar la equidad desde un planteamiento democrático, inclusivo y comprometido con la justicia social.
\end{abstract}

Palabras clave: fondos de conocimiento; interculturalidad; inclusión educativa; COVID-19; alumnado vulnerable.

\section{Resumo}

Actualmente, os sistemas educativos mundiais estão a atravessar um período de incerteza causado pela eclosão de uma crise sócio-sanitária causada pela COVID-19, que levou à implementação de toda uma gama de soluções educativas numa modalidade híbrida para assegurar a continuidade educativa nas melhores condições possíveis. Esta situação sem precedentes teve um grande impacto em todos os agentes educacionais, afectando significativamente os estudantes mais vulneráveis.

O objectivo deste documento é analisar os efeitos da pandemia causada pela COVID-19 nos estudantes imigrantes vulneráveis, reflectir sobre os Fundos do Conhecimento como instrumentos essenciais para tornar as minorias étnicas visíveis e tentar investigar, de forma crítica e reflexiva, as acções que podem ser decisivas para garantir a equidade a partir de uma abordagem democrática, inclusiva e comprometida com a justiça social.

Palavras-chave: fundos de conhecimento; interculturalidade; inclusão educacional; COVID-19; estudantes vulneráveis.

\footnotetext{
${ }^{1}$ Universidad de Málaga | E-mail: mjalcaladelolmo@uma.es

${ }^{2}$ Universidad de Málaga |E-mail: juanleiva@uma.es
} 


\section{Introducción}

Vivimos momentos históricos, sociales, culturales, tecnológicos, políticos y de salud francamente complejos y de gran incertidumbre. Parafraseando a Bauman (2015) podríamos afirmar que la modernidad ya no es incluso líquida, sino gaseosa, difícilmente estable, predecible y calificable. Es más, hablamos de una época culturalmente definida por la postmodernidad, la influencia de las redes sociales, la proliferación de las fake news, la imparable digitalización de la educación y de las relaciones interpersonales, así como un crecimiento de la influencia del Big Data y una globalización tecnológica que hace de nuestro mundo un lugar culturalmente más homogéneo determinado por la emergencia de una cultura tecnológica que pone de relieve las contradicciones de la globalización.

Igualmente, asistimos perplejos a una tendencia muy significativa en materia social, moral y política, una polarización que es fruto de décadas de un exacerbado neoliberalismo que ha puesto y sigue poniendo en tela de juicio la sostenibilidad ambiental y los derechos humanos, como retos que continúan siendo determinantes en la realidad actual. Como respuesta, observamos ahora mismo con estupor los ataques sin precedentes hacia la democracia liberal por parte de postulados extremistas y totalitarios, bien de corte social-populista como nacional-populista. Recientemente, podemos recordar que el día 6 de enero de 2021, en EE.UU, hubo una especie de insurrección de grupos y personas extremas para tratar de impedir la votación en el propio Capitolio en la elección del presidente Biden. Pero ¿por qué decimos esto en un artículo eminentemente social y pedagógico? Porque debemos tener claro que las respuestas sociales y educativas de interculturalidad son la mejor "vacuna" para la prevención y el abordaje integral de este tipo de postulados y valores antidemocráticos que no solo cuestionan la democracia liberal y el estado social y democrático de derecho, sino que en ambos polos (de ideología extrema de ultraderecha y ultraizquierda), se pretende utilizar la escuela, los contextos sociales y comunitarios como espacios para el adoctrinamiento, la sumisión, el incremento de las desigualdades y la discriminación, el fomento del conformismo y la ausencia del pensamiento crítico y libre. En coherencia con los argumentos anteriores, podemos poner de manifiesto que la interculturalidad necesita en estos tiempos de COVID-19 una reformulación más inclusiva sobre sus caminos de respeto a la diversidad y proyecciones didácticas, empleando para ello nuevas herramientas y dispositivos digitales capaces de empoderar a familias, estudiantes, profesorado, y comunidades, proporcionando los recursos que resultan indispensables 
para trabajar la resiliencia y superar en las mejores condiciones posibles estos momentos de adversidad ante los que nos encontramos inmersos.

Ante todo ello, y, especialmente por la proliferación de actitudes racistas, violentas, populistas y antidemócratas, y la necesidad de reducir y erradicar las desigualdades en el ámbito educativo, resulta imprescindible repensar y re-generar nuevos marcos de comprensión y de acción social y educativa. En este sentido, los fondos de conocimiento se configuran, a nuestro juicio, como un excelente marco teórico-práctico de carácter democrático e inclusivo que puede facilitar iniciativas sociales y pedagógicas para transformar la realidad de los contextos educativos, revitalizando el trabajo educativo, social y comunitario para buscar el mejor y mayor impacto democrático en las comunidades escolares que son comunidades locales.

Precisamente, en este artículo vamos a abordar los efectos de la COVID-19 en el alumnado inmigrante en situación de vulnerabilidad porque constituye uno de los grupos cuyos apoyos educativos requieren una mayor clarificación de la situación social, comunitaria y pedagógica. El objetivo es indagar de forma crítica y reflexiva y siempre desde un enfoque pedagógico democrático e inclusivo, los dilemas y paradojas que nos pueden ayudar a comprender los efectos de la Pandemia en estudiantes inmigrantes en situación de riesgo de exclusión social.

\section{Fondos de conocimiento y Educación Intercultural}

En los últimos tiempos en el ámbito educativo estamos asistiendo a una normativización excesiva de la vida escolar. No podemos estar en desacuerdo con los necesarios procesos de regulación normativa, pero el alto grado de concreción y traducción pedagógica puede suponer una merma en la autonomía educativa de los centros escolares. Así, nos parece muy interesante el desarrollo de propuestas educativas interculturales dentro del paradigma de la educación inclusiva, el modelo de la educación para todos y la búsqueda de nuevas fórmulas de innovación educativa para reducir y/o eliminar cualquier atisbo de desigualdad en el escenario educativo (Milner, 2017). Dicho esto, irrumpir en los contextos educativos desde una mirada intercultural e inclusiva supone atender a claves de reconocimiento de la diversidad cultural, justicia social y educativa (Goodwin \& Darity, 2019; Sleeter, 2012).

Igualmente, supone también reconceptualizar que existe una cultura escolar que responde a los intereses, normas y códigos semánticos establecidos de forma más o menos consciente por los distintos agentes educativos, aunque obviamente influenciados por 
elementos sociales y culturales que hoy día cobran gran importancia. En este sentido, más allá de los posicionamientos y de las propias trayectorias personales y profesionales de los docentes, existen elementos conceptuales macro y micro que determinan los procesos formativos. Si bien las propuestas para el desarrollo de la interculturalidad están en las agendas de muchas instituciones escolares, es importante detenernos en algunos elementos que actualmente determinan el perfil, carácter, impronta y orientación de la educación intercultural (Aguaded-Ramírez, 2017). Precisamente, en un trabajo anterior de Leiva (2017), se planteaba la idea de que los procesos pedagógicos requieren una revisión en términos de una interculturalización curricular, lo que supone ser consciente de determinados factores o parámetros que pueden negar o dificultar, o bien posibilitar esta profunda reflexión pedagógica para la práctica escolar. En primer lugar, la minimización de lo cultural como factor pedagógico, que significa que hay equipos directivos, docentes u otros agentes educativos (familias, etc...) que no contemplen la diversidad cultural como un elemento sociocultural de interés y relevancia para su tratamiento educativo, e incluso, la negación de la diversidad. En segundo lugar, la rutinización de la interculturalidad como moda pasajera, que en la práctica supone que todavía persiste en un buen número de centros escolares un enfoque educativo más bien romántico y/o folclorista de la diversidad cultural. En tercer lugar, la compasión como talante pedagógico ante la diversidad cultural, manifestada en algunos docentes y familias con actitudes compasivas, que no hacen sino resurgir la caridad (más que solidaridad), lo que no ayuda al cambio y a la transformación de mentalidades en pro de una educación intercultural e inclusiva que promueva la dignidad, la libertad, la alteridad, la justifica social, la legitimación y la solidaridad entre las personas, las familias y el propio alumnado (Sleeter, 2017).

Todo ello nos lleva a cuestionarnos la necesidad de asumir una nueva mirada epistemológica acerca del desarrollo de la interculturalidad en los centros educativos, de tal forma que podemos y debemos poner el foco de atención en los Fondos de Conocimiento. Si bien estos surgen en el marco de algunos trabajos de antropología y psicología cultural en la década de los 90 en EE.UU. como un modo de explicitar y evidenciar los saberes culturales y educativos en las familias mexicanas de origen inmigrante en el estado de Arizona (Moll \& González, 1994), hoy nos interesa como palanca conceptual de cambio y estrategia para transformar la perspectiva deficitaria ante los nuevos problemas en el marco de la COVID-19 y los cambios y dificultades en la atención educativa de los estudiantes más vulnerables desde un enfoque de inclusividad 
(Esteban-Guitart, Oller, \& Vila, 2012; Esteban-Guitart \& Saubich, 2013; Esteban-Guitart \& Vila, 2013; Esteban-Guitart \& Llopart, 2017).

La educación está abordando en estos años (2020 y 2021) el mayor de los cambios en la historia de la educación en la humanidad, especialmente por la necesidad emergente de promover activamente una mayor virtualización de los procesos de enseñanza y aprendizaje, por un lado, y no obviar de que estos procesos incorporen dispositivos, recursos y estrategias educativas de innovación e inclusión educativa, por otro lado. Así, podemos inscribir aquí la necesidad de no olvidar la educación en valores, la necesidad de educar en y para la diversidad en momentos en los que crece la intolerancia, la discriminación, el racismo y la xenofobia (Milner, 2015). En este sentido, debemos expresar que no se trata de cambiar aspectos menores en el diseño o planificación didáctica, sino de incidir en una mayor humanización y sostenibilidad de los procesos educativos ahora que la tecnología está influyendo de forma decisiva en los modos de comunicarnos, de interactuar y de relacionarnos como seres humanos, y, también, como agentes educativos tanto con el resto de personas como con nuestro medio ambiente (Alcalá del Olmo, Santos-Villaba, Leiva \& Matas, 2020; Fantini, 2020; Leiva, 2020; Pareja de Vicente, Leiva, \& Matas, 2020).

No se trata, en ningún caso, de una propuesta militante dogmática de corte totalitario o populista, sino de una filosofía que busca respetar y aprender de la diversidad como valor auténticamente humano para generar una conciencia educativa más inclusiva y democrática. No se trata de enfrentar o de generar grupos de poder en la escuela, sino de transformar la educación en mayor humanidad en términos de favorecer la inclusión educativa y la máxima calidad para todo el alumnado y sus familias.

Dentro de lo que venimos argumentando, los Fondos de Conocimiento vienen a suponer un elemento clave en el reconocimiento de la visibilidad de las minorías étnicas, de las diferencias culturales que debe ser lo común y lo que genera posibilidades de interdependencia y riqueza educativa y social. Podríamos definirlos como el conjunto de saberes y competencias, culturalmente cultivados e históricamente acumulados a lo largo del tiempo que resultan fundamentales para el funcionamiento y dinámicas del bienestar, tanto individual como familiar y colectivo (González, Moll, \& Amanti, 2005). Esto significa que los Fondos de Conocimiento se configuran como una forma explícita de denominación de un conjunto interdependiente de saberes y pautas de interacción que se hacen visibles fundamentalmente en la ecología familiar y comunitaria. ¿Qué supone esto en la práctica educativa? Que estos saberes entendidos como códigos semánticos o de 
funcionamiento individual que se derivan de la familia no deben entenderse como una socialización divergente o disfuncional, sino que resulta legítima dentro de la amplia amalgama de diversidades y diferencias sociales y culturales.

Ni qué decir tiene que los Fondos de Conocimiento, conceptualmente, vienen a intentar reconceptualizar una narrativa que debe hacerse explícita en los centros educativos, esto es, se debe cambiar el lenguaje para que sea más inclusivo y nos ayude a transformar el contexto y las propias realidades de desigualdad y desafección (Anderson \& Macleroy, 2017; Chen, Carger, \& Smith, 2017). También se trata de que la escuela ha priorizado siempre una cultura escolar mesocrática, donde supuestamente había un currículum aséptico y aparentemente neutral (Ríos-Aguilar, Kiyama, Gravitt, \& Moll, 2011). Hoy nadie pone en duda la necesidad de posibilitar la construcción participativa, democrática e inclusiva del currículum donde los profesionales de la educación son guías, facilitadores, mediadores y promotores del aprendizaje en un sistema que debe perseguir la calidad y la excelencia. Pero la excelencia no entendida dentro de parámetros restrictivos o reduccionistas de resultados de rendimiento académico o ranking de niveles de adquisición competencial, sino en términos de integralidad del derecho de la educación de calidad, el de una educación inclusiva e intercultural que ayude a todo el alumnado y a sus familias a un mayor desarrollo, bienestar y empoderamiento.

Plantear el discurso y la práctica educativa como elementos de negación al otro derivan siempre en dogmatismos y en planteamientos populistas o totalitarios que niegan la identidad de los saberes que existen en toda ecología familiar y social (Varga-Dobai, 2018). Resulta necesario indagar de forma reflexiva y participativa en una narrativa pacífica, respetuosa con la diversidad, la libertad y la crítica libre. De este modo, el problema o "hándicap" no se sitúa en las familias, en los estudiantes de comunidades minorizadas, sino en el etnocentrismo de una hegemonía que tiende a perpetuar y a legitimar una relación jerárquica - injusta - de saberes y formas de interactuar, de vivir, de sentir y aprender (Cortina \& Earl, 2020; Lorenzo, Míguez, \& Cernadas, 2020).

Desde una perspectiva educativa, se trata de rechazar en la escuela cualquier actitud de discriminación, de rechazo a la diversidad o de invisibilidad de las situaciones que deben ser corregidas desde el punto de vista de las necesarias acciones educativas inclusivas y de interculturalidad (Louzao, Francos, \& Verdeja, 2020). El etnocentrismo y el racismo, o cualquier otra forma de intolerancia no puede caber en una institución que no solamente debe enseñar e inculcar valores democráticos, sino que debe practicarlos para propiciar el desarrollo personal y colectivo en libertad, justicia social e inclusión 
(Sherfinski, Hayes, Zhang, \& Jalalifard, 2020). El encuentro, la acogida mutua y el acercamiento cultural, emocional y moral se vislumbra como esencial para que la interculturalidad tenga una consonancia y dinámica autentica de inclusión educativa (Alharbi, Tan, \& Lo, 2020; Cho \& Yi, 2020; Morales, 2019). El currículum escolar no puede ser estandarizado o de "talla única" (Pérez-Gónez, 2012), sino todo lo contrario, recogiendo los Fondos de Conocimiento de todos los agentes educativos, y especialmente de aquellas familias y estudiantes que proceden de contextos que han sido marginados o que se encuentran en riesgo de exclusión y alta vulnerabilidad (Varga-Dobai, 2018).

La jerarquía de valores y de saberes, tanto de corte experiencial como académica debe atender al marco constitucional, de respeto a los derechos humanos, a los objetivos de desarrollo sostenible que nos indica la ONU y la UNESCO, así como otras instancias internacionales que son valedoras de la igualdad y la libertad (Milner, 2011). En un mundo interconectado, las escuelas conforman espacios cruciales para que los más jóvenes se conecten a la democracia, el respeto a la diversidad y la inclusión sin caer en ningún momento en prácticas excluyentes o populistas, sino todo lo contrario, en opciones curriculares y democráticas que sean científicas, racionales, rigurosas y que procuren la interculturalidad y la inclusión en todo momento (McBride, Bellamy, \& Knoester, 2020).

\section{El desarrollo de la interculturalidad en tiempos de pandemia}

La actual sociedad está sujeta a cambios constantes y vertiginosos, generados por la globalización, los avances científicos y tecnológicos, la complejidad de las estructuras sociales, la gran incidencia de los medios de comunicación, la interculturalidad, la marcada heterogeneidad social y las modificaciones laborales, entre otras variables.

Unidos a estos cambios, nos encontramos ante una crisis internacional provocada por el virus de la COVID-19, que afecta, tanto al llamado Estado del Bienestar, como a las esferas económicas, sociales y educativas. Estamos ante un nuevo escenario en el que, además de ser indispensable el replanteamiento de los "modus vivendi" actuales, precisamos, desde una perspectiva pedagógica e inclusiva, nuevos formatos de enseñanza y de aprendizaje, resultando los paradigmas educativos piezas esenciales en el engranaje de una cadena que busca el diseño de procesos educativos capaces de ofrecer respuesta a las nuevas realidades y circunstancias que nos rodean en la actualidad.

La Organización de Naciones Unidas para la Educación, la Ciencia y la Cultura, nos muestra el impacto de la pandemia de la COVID-19 en la educación en el mundo, encargándose este Organismo de apoyar a los países en sus esfuerzos por paliar el efecto 
del cierre de escuelas, especialmente en aquellas comunidades más desfavorecidas y vulnerables, tratando de ofrecer directrices pedagógicas con las que facilitar la continuidad de la educación para todos y todas a través de un aprendizaje híbrido y alejado de la plena presencialidad, sin que ello afecte a la calidad de la formación proporcionada. En este sentido, el Informe de Seguimiento de la Educación en el Mundo 2020 (Global Education Monitoring Report Team, 2020), bajo el título "Inclusión y Educación: Todos sin excepción", refleja las dimensiones de discriminación existentes en diversas partes del mundo por razones de género, lugar de residencia, status socio-económico, diversidad funcional, etnia, idioma, migración, orientación sexual, religión, creencias y actitudes, constituyendo todas ellas variables que afectan de forma muy significativa a la posibilidad de los niños y niñas a recibir una educación de calidad en igualdad de condiciones y de oportunidades.

Con el objetivo de superar esta situación, la UNESCO se encarga de colaborar con los ministerios de educación de los diferentes países afectados por la COVID-19 para garantizar la continuidad del aprendizaje de los niños y jóvenes, ofreciendo, entre sus respuestas globales, la llamada Coalición Mundial para la Educación (Unesco, 2020a), con el firme propósito de ayudar a los países a ofrecer las mejores condiciones para el aprendizaje y llegar a aquellos estudiantes que puedan encontrarse en una situación de mayor riesgo y fragilidad social. Entre algunas de las medidas planteadas, destaca la necesidad de proporcionar soluciones inclusivas de aprendizaje a distancia que permitan atender en las mejores condiciones posibles a aquellos estudiantes más vulnerables en la situación de emergencia socio-sanitaria ante la que nos encontramos, como forma de contribuir a la conformación de sistemas educativos resilientes e inclusivos.

En coherencia con los argumentos anteriores, nos parece importante reflexionar en torno al desarrollo de la interculturalidad en estos momentos de pandemia y desazón en los que nos encontramos, como forma de subrayar la necesaria toma de conciencia ante lo indispensable que resulta proporcionar una óptima respuesta al alumnado procedente de otros países y culturas, resultando, en buena parte de los casos, el colectivo más azotado en estos momentos.

En primer lugar, es imprescindible poner de relieve que los centros escolares conforman espacios privilegiados para atender a la dimensión intercultural y facilitar su abordaje transversal desde los tejidos curriculares, entendida la educación intercultural desde un enfoque antirracista y antropocéntrico, destinada a combatir toda forma de discriminación y de desigualdad (Dietz \& Mateos, 2020; Leiva, 2020; Louzao, 2019; 
Louzao, Francos, \& Verdeja, 2020). Para atender a la diversidad cultural, el profesorado cuenta con diversas dificultades, que, en realidad, se han visto notablemente acrecentadas en estos momentos de pandemia en los que nos encontramos, en tanto que, los formatos de docencia virtual hacen aún más complejo atender a los diferentes ritmos de aprendizaje de este alumnado y su diversidad lingüística, careciendo de los recursos necesarios con los que trabajar con plena eficacia en contextos multiculturales. En relación con los estudiantes culturalmente diversos, la pandemia no ha hecho sino incrementar sus dificultades para acceder en condiciones adecuadas al currículum, de la misma forma que, al desaparecer los contactos físicos con su grupo de iguales, buena parte de ellos y ellas han desarrollado desfases curriculares que, en muchos casos, se superaban presencialmente en situaciones normalizadas mediante experiencias de aprendizaje compartido. Las familias, al mismo tiempo, han tenido que hacer frente a situaciones de aislamiento relacional y emocional, de la misma forma que se han visto ante la necesidad de ofrecer acompañamiento educativo a sus hijos e hijas en la realización de las tareas académicas, con las consiguientes dificultades idiomáticas, cognitivas en muchos casos y relativas a la conciliación.

Diferentes estudios que se han centrado en analizar el impacto del confinamiento en el alumnado más vulnerable (Bayona, Domingo, \& Menacho, 2020; FernándezRodrigo, 2020; Von- Braun, Zamagni, \& Sánchez-Sorondo, 2020) en el que podemos ubicar al culturalmente heterogéneo, han conseguido reflejar que la crisis socio-sanitaria en la que nos encontramos ha hecho incrementar de forma significativa la inequidad, evidenciándose desigualdades de equipamiento tecnológico y de preparación entre familias, centros educativos y profesorado, extendiendo las brechas digitales de aprendizaje que ya existían antes de estos momentos de crisis, e incluso generando otras nuevas (Iglesias, González-Patiño, Lalueza, \& Esteban-Guitart, 2020). Desde esta perspectiva, ha podido evidenciarse que el nivel adquisitivo y de renta de las familias condiciona de forma muy evidente el acceso a los recursos digitales y el acceso referido a las horas y momentos dedicados al trabajo escolar, de la misma forma que el nivel formativo de los progenitores se relaciona directamente con las estrategias puestas en marcha para apoyar la realización de las tareas escolares (Tarabini, 2020), dedicando además los niños y niñas y el colectivo adolescente el tiempo transcurrido durante el encierro en los domicilios a actividades muy diversas en función del capital instructivo de las familias (Bayona, Domingo, \& Menacho, 2020). 
El conjunto de estos estudios ofrece evidencias que ayudan a admitir que el confinamiento en momentos de pandemia ha generado efectos especialmente negativos en estudiantes procedentes de entornos vulnerables, influyendo todo ello en cuestiones referentes a la justicia social y la equidad (Echeita, 2020).

Para tratar de paliar este tipo de situaciones, es necesario repensar de qué forma equilibrar las pérdidas tanto de tipo emocional, como cognitivo y social que se hayan generado en los momentos confinamiento y en los formatos de docencia virtual, buscando articular medidas de ajuste y concreción curricular, orientaciones sobre evaluación final en cada de una de las disciplinas formativas, programas de refuerzo educativo y provisión de recursos, tanto humanos como materiales que permitan ofrecer una atención educativa de calidad y excelencia al alumnado más vulnerable.

En coherencia con lo anterior, el Diseño Universal de Aprendizaje (DUA) se nos muestra como una estrategia eficaz ante estos nuevos escenarios y realidades educativas, en tanto que postula una educación favorecedora de todas y cada una de las personas (Villa-Fernández \& Martín-Gutiérrez, 2020), apostando por hacer realidad una educación verdaderamente inclusiva. No es baladí reconocer, por tanto, que constituye un instrumento fundamental mediante el que diseñar el currículum, buscando que todos y todas las alumnas lleguen a adquirir habilidades, conocimientos y motivación suficiente con la que construir aprendizajes relevantes y significativos.

\section{Dilemas y paradojas ante los efectos de la COVID-19 en situación de vulnerabilidad}

La repentina aparición en China de la COVID-19 en el mes de diciembre de 2019, con su consiguiente expansión a lo largo de todo el globo terráqueo en los meses siguientes, ha representado, atendiendo a su alcance y gravedad, un desafío global sin precedentes. A la gran cantidad de pérdidas humanas, pueden sumarse los efectos perversos que esta crisis socio-sanitaria está originando en la economía de casi todos los países (Di Franco et al., 2020), del mismo modo que ha podido constatarse su gran influencia en los espacios educativos de todo el Planeta (Bonilla, 2020).

Dentro de las primeras medidas adoptadas para frenar la propagación de la cadena vírica, destaca el cierre y suspensión temporal de la docencia presencial en las instituciones educativas en todos los niveles, lo que afectaría prácticamente a 1.520 millones de estudiantes distribuidos en distintas partes del mundo (Unesco, 2020b). Todo ello dio lugar a una situación de confinamiento cuyos fines iban destinados a salvaguardar la salud de todos y todas, si bien generó importantes repercusiones en los diversos 
miembros de la comunidad educativa. En concreto, hubo que asumir el importante y repentino desafío de recurrir a la virtualidad, a partir de formas alternativas de enseñanza $\mathrm{y}$ aprendizaje fundamentadas en el uso de herramientas tecnológicas con las que garantizar la continuidad pedagógica en las mejores condiciones posibles (Avello, 2020).

Durante esta situación de cierre temporal de las instituciones educativas, la emergencia sanitaria contribuyó a reflejar gran parte de fragilidades y carencias tanto relativas a la disponibilidad de los recursos digitales que permitieran seguir construyendo conocimientos, como a la preparación docente para trabajar en estos nuevos escenarios. El profesorado, en este sentido, no disponía de la formación necesaria con la que llegar a extraer al máximo las potencialidades de los recursos digitales, viéndose obligado a desarrollar un comportamiento autodidacta que le permitiera, no solo redefinir su praxis docente como forma de seguir en contacto con su alumnado, sino asegurar la adquisición de competencias y la construcción de aprendizajes funcionales relacionados con los aspectos esenciales de cada una de las disciplinas impartidas. De la misma forma, se encontró ante la inestimable necesidad de desarrollar funciones de acompañamiento y tutorización de un alumnado repleto de incertidumbres e inseguridades ante un escenario educativo nunca antes conocido (Sanz, Sáinz, \& Capilla, 2020), en el que proliferaba la soledad emocional y el desarraigo ante la desaparición de relaciones interpersonales con un grupo de iguales con el que, hasta el momento, se compartían inquietudes, vivencias y se construía, en muchos casos, de forma cooperativa el conocimiento. Esta situación se agravó particularmente en el alumnado más vulnerable, en el que las consecuencias derivadas de la brecha digital se hacían aún más evidentes (Murillo \& Duk, 2020), precisando por ello una mayor atención y sensibilidad para poder asegurar la adquisición de aprendizajes y la permanencia en el proceso formativo en igualdad de condiciones.

En efecto, han sido numerosos los estudiantes que, atendiendo a complejas situaciones económicas, personales y de salud, han presentado serias dificultades para disponer de un eficiente acceso a Internet, bien sea porque no pudieron permitirse un equipo informático, o bien porque sus lugares de residencia contaban con una deficitaria conectividad. De la misma forma, los estudiantes con discapacidad también figuraron entre los más afectados, al tratarse de un colectivo que requiere medidas educativas específicas e instrumentos de enseñanza que no siempre están disponibles en la modalidad de una educación a distancia, encontrándose así los centros educativos ante el reto de garantizar una formación virtual, sostenible e inclusiva, capaz de atender de la mejor forma posible a un alumnado potencialmente heterogéneo. 
En un contexto de crisis sanitaria como al que nos estamos refiriendo, por tanto, es importante mencionar la perspectiva de la equidad (Echeita, 2020; Hueso, 2020; Rodríguez, 2020), no solo ante la existencia de brechas digitales, sino también ante el cuestionamiento acerca de la potencialidad de los centros educativos para configurarse en espacios saludables, seguros e inclusivos, evidenciándose marcadas diferencias entre instituciones educativas pertenecientes a diversas partes del mundo, lo que ha llevado a constatar que el logro de la equidad y, con él, la atención educativa brindada al alumnado más vulnerable, continúa siendo uno de los mayores retos a los que nos enfrentamos en la actualidad (Echeita, 2020; Valenzuela, 2020).

La Agenda 2030 para el Desarrollo Sostenible, en consonancia con la disyuntiva anterior, plantea la necesidad de adoptar una perspectiva holística y transversal que abarque los grandes retos vitales tanto del Planeta como de todas las personas (Unesco, 2016, 2017), presentando los Objetivos del Desarrollo Sostenible una relación de interdependencia indisoluble. Trasladar estos planteamientos a los centros educativos supone percibir en ellos instituciones comprometidas con los desafíos delimitados en la Agenda, concentrando los esfuerzos en hacer realidad una formación inclusiva, con la que, además de garantizar una formación de excelencia en términos de rectitud, pueda atenderse a la coyuntura socio-sanitaria en la que estamos inmersos y romper las brechas existentes, relacionadas, no solo con variables socioeconómicas, sino también de género, digitales y de conocimiento.

Y es que la crisis en la que nos encontramos inmersos, puede servir de pretexto para valorar detenidamente y de forma rigurosa las carencias principales de nuestros sistemas educativos y los consiguientes desafíos que los profesionales de la educación debemos asumir en el mundo interdependiente y global que nos rodea. La inédita situación en la que estamos inmersos, así pues, ha puesto de relieve lo indispensable que resulta contar con una buena planificación (Cabrera, 2020), diseñando espacios de aprendizaje y de evaluación lo suficientemente flexibles con los que conceder una respuesta eficiente a las situaciones sobrevenidas que puedan desencadenarse.

Situando la mirada en un análisis de las repercusiones fundamentales derivadas de la pandemia originada por la COVID-19 en los diversos agentes educativos, en la comunidad científica nacional e internacional han proliferado diferentes investigaciones que han tratado de valorar de forma detenida y analítica la perplejidad a la que se vieron sometidos tanto estudiantes como familias y docentes de todo el mundo ante la crisis 
sanitaria vivida (Bayham \& Fenichel, 2020; Crosier \& Vasilieiou, 2020; Giroux, RiveraVargas, \& Passeron, 2020; Osmond-Johnson, Campbell, \& Pollock, 2020).

En el caso del profesorado, los estudios ponen de relieve la situación de desconcierto a la que tuvo que enfrentarse este sector profesional para realizar un ajuste emergente, transitando de forma vertiginosa de una docencia presencial a una docencia en línea, manejando herramientas tecnológicas que hasta ese momento eran desconocidas en muchos casos, resolviendo dudas a través de foros, buscando respuestas de forma autodidacta, en un escenario repleto de interrogantes acerca de cómo trabajar desde la distancia para asegurar la calidad de la formación proporcionada.

Refiriéndonos a los estudiantes, los estudios aluden a los efectos perversos de la ruptura de la cotidianeidad, las marcadas dosis de ansiedad e incertidumbre ante una situación nunca antes vivida y la sobrecarga de tareas académicas solicitadas por ciertos docentes que decidieron "compensar" la ausencia de clases presenciales con una ingente cantidad de trabajos analíticos y de indagación con los que cubrir los temas que no podían abordarse en el espacio docente presencial de las disciplinas. A todo ello puede sumarse la necesidad de presentar un mayor conocimiento de las herramientas digitales, una mayor comprensión lectora, ajustes en los horarios para conciliar la vida personal con la académica y una mayor responsabilidad y autonomía pedagógica en general. En este sentido, las investigaciones advierten acerca del necesario desarrollo de un autoaprendizaje tecnológico, competencias digitales y socioemocionales y manejo eficiente de entornos virtuales de aprendizaje.

En relación con las familias, cabe destacar la obligación repentina de hacerse cargo de sus descendientes a lo largo de toda la jornada, buscando conciliar de la mejor forma posible la vida personal con la laboral y asumiendo responsabilidades educativas en orden a asegurar la implicación de sus hijos e hijas en procesos educativos virtuales.

\section{Conclusiones}

Nos encontramos en un momento de gran incertidumbre, en el que resulta indispensable aprender de la experiencia, perfilar propuestas pedagógicas preventivas y de gestión del riesgo y desarrollar una capacidad de empoderamiento y de resiliencia con la que afrontar de la mejor forma posible las crisis que lleguen a desencadenarse. Son muchos los retos que debe afrontar nuestro actual sistema educativo, siendo uno de los principales paliar los efectos devastadores de la desigualdad de oportunidades educativas, prestando especial atención al alumnado más vulnerable, haciendo realidad una 
pedagogía inclusiva que permita educar en la resiliencia y en la superación y fortalecimiento ante la adversidad.

Lo que estamos viviendo, desde un punto de vista social y pedagógico, es una auténtica revolución. Los cambios metodológicos, didácticos y tecnológicos que se están generando han llegado para quedarse y no tendrán un carácter coyuntural, especialmente en términos de una mayor virtualización y digitalización de los procesos de enseñanza y aprendizaje. Ahora bien, no es menos cierto que la socialización humana requiere de elementos de presencialidad, estos cambios acaecidos van a promover de forma activa la expansión del blended learning, a la vez que el fomento de nuevas miradas pedagógicas sobre el significado mismo de qué es la educación, al servicio de qué tipo de sociedad y qué tipo de formación tiene un carácter más aplicado y relacionado con el desarrollo integral de las personas y la sociedad en su conjunto.

Igualmente, los Fondos de Conocimiento van a ser un revulsivo para la praxis educativa intercultural, especialmente en estos momentos en que muchos centros escolares pueden y deben convertirse en referencia cultural clave para sus comunidades locales. La implementación de una mayor comunicación digital requiere de programas y talleres específicos de alfabetización para familias y estudiantes, así como un compromiso más decidido e ineludible por la generación de una cultura de la diversidad que incida en la inclusión y los valores democráticos de justicia social y libertad. Todas aquellas propuestas didácticas que activen la participación y dinamicen el protagonismo y la visibilidad de todos los significados culturales, familiares y de diversidad cultural que permanecen ocultos, no reconocidos o invisibles, será fundamentales en el desarrollo de procesos educativos interculturales, trampolín para aprender y vivir en armonía, concordia y paz.

Los estudiantes de origen inmigrante y todos aquellos pertenecientes a minorías sociales y étnicas no pueden quedarse atrás en estos momentos, por lo que la escuela es y debe ser el principal mecanismo social y comunitario que puede servir de dique de contención, de resiliencia y empoderamiento para poder avanzar en nuevos pasos y caminos de interculturalidad, inclusión y bienestar.

\section{Referencias bibliográficas}

Aguaded-Ramírez, E. (2017). Smart city and Intercultural Education. ProcediaSocial and Behavioral Sciences, 326-333. https://doi.org/10.1016/j.sbspro.2017.02.010 
Alcalá del Olmo, M. J., Santos-Villaba, M.J. Leiva, J., \& Matas, A. (2020). Sostenibilidad Curricular: Una Mirada desde las Aportaciones del Profesorado de la Universidad de Málaga. Revista Internacional de Educación para la Justicia Social, 9(2), 309-326. https://doi.org/10.15366/riejs2020.9.2.015

Alharbi, M., Tan, Y. S. M., \& Lo, C. S. O. (2020). Foodways, community, and filmmaking: a case study of funds of knowledge in higher education. Higher Education Pedagogies, 5(1), 310-323. https://doi.org/10.1080/23752696.2020.1841569

Anderson, J., \& Macleroy, V. (2017). Connecting worlds: interculturality, identity and multilingual digital stories in the making. Language and Intercultural Communication, 17(4), 494-517. https://doi.org/10.1080/14708477.2017.1375592

Avello, I. Q. (2020). Covid-19 y Cierre de Universidades ¿Preparados para una Educación a Distancia de Calidad? Revista Internacional de Educación para la Justicia Social, 9(3), 1-11.

Bauman, Z. (2015). Modernidad líquida. Fondo de cultura económica.

Bayham, J. \& Fenichel, E. (2020). Impact of school closures for COVID-19 on the US healthcare workforce and net mortality: a modelling study. The Lancet Public Health, 5(5), 271-278. https://doi.org/10.1016/s2468-2667(20)30082-7

Bayona, J., Domingo, A., \& Menacho, T. (2020) Trayectorias migratorias y fracaso escolar de los alumnos inmigrados y descendientes de migrantes en Cataluña. Revista Internacional de Sociología, 78 (1). https://doi.org/10.3989/ris.2020.78.1.18.107

Bonilla, J.A. (2020). Las dos caras de la educación en el COVID-19. CienciAmérica, 9(2), 89-98. http://dx.doi.org/10.33210/ca.v9i2.294

Cabrera, L. (2020). Efectos del coronavirus en el sistema de enseñanza: aumenta la desigualdad de oportunidades educativas en España. Revista de Sociología de la Educación, RASE, 13(2), 114-139. http://dx.doi.org/10.7203/RASE.13.2.17125

Crosier, D. \& Vasilieiou, N. (2020). How should students be admitted to higher education this year? Eurydice. European Commission. Recuperado de https://eacea.ec.europa.eu/national-policies/eurydice/content/how-should-stu-dents-beadmitted-higher-education-year_en

Chen, Y., Carger, C. L., \& Smith, T. J. (2017). Mobile-assisted narrative writing practice for young English language learners from a funds of knowledge approach. Language Learning \& Technology, 21(1), 28-41. https://dx.doi.org/10125/44594

Cho, S., \& Yi, Y. (2020). Funds of knowledge and cultural capital: Working toward diversity and equity of knowledges. Applied Linguistics, 41(5), 810-815. https://doi.org/10.1093/applin/amy062

Cortina, R., \& Earl, A. (2020). Embracing interculturality and Indigenous knowledge in Latin American higher education. Compare: A Journal of Comparative and International Education, 1-18. https://doi.org/10.1080/03057925.2020.1766350

Dietz, G. \& Mateos, L.S. (2020): Mexican intercultural education in times of COVID-19 pandemic, Intercultural Education, https://dx.doi.org/10.1080/14675986.2020.1843895

Di Franco, M. G., Trainer, J. A., Bazán, S., \& Porta, L. G. (2020). Concatenaciones fronterizas: pedagogías, oportunidades, mundos sensibles y COVID-19. Praxis Educativa, 24(2), 1-18. https://dx.doi.org/10.19137/praxiseducativa-2020-240203 
Echeita, G. (2020). La Pandemia del Covid-19. ¿Una Oportunidad para Pensar en Cómo Hacer más Inclusivos Nuestros Sistemas Educativos? Revista Internacional de Educación para la Justicia Social, 9(1), 7-16.

Esteban-Guitart, M., Oller, J., \& Vila, I. (2012). Vinculando escuela, familia y comunidad a través de los fondos de conocimiento e identidad. Un estudio de caso con una familia de origen marroquí. Revista de Investigación en Educación, 10(2), 21-34.

Esteban-Guitart, M., \& Saubich, X. (2013). La práctica educativa desde la perspectiva de los fondos de conocimiento e identidad. Teoría de la Educación. Revista Interuniversitaria, 25(2), 189-211. https://doi.org/10.14201/11583

Esteban-Guitart, M., \& Vila, I. (2013). La modificación de las creencias sobre las familias de origen inmigrante por parte de docentes después de participar en el proyecto educativo "fondos de conocimiento". Cultura y educación, 25(2), 241-254. https://doi.org/ 10.1174/113564013806631282

Esteban-Guitart, M., \& Llopart, M. (2017). La inclusión educativa a través de la aproximación de los fondos de conocimiento e identidad. Revista de educación inclusiva, 9(3), 145-157.

Fantini, A. E. (2020). Reconceptualizing intercultural communicative competence: A multinational perspective. Research in Comparative and International Education, 15(1), 52-61. https://doi.org/10.1177/1745499920901948

Fernández-Rodrigo, L. (2020). Alumnado que no sigue las actividades educativas: El caso de una escuela de alta complejidad durante el confinamiento por COVID-19. Sociedad e Infancias, 4,185-288. http://dx.doi.org/10.5209/soci.69266

Gay, G. (2010). Culturally responsive teaching: Theory, research, and practice. Teachers College Press.

Giroux, H., Rivera-Vargas, P., \& Passeron, E. (2020). Pedagogía Pandémica. Reproducción Funcional o Educación Antihegemónica. Revista Internacional de Educación para la Justicia Social, 9(3). Recuperado de https://revistas.uam.es/riejs/article/view/12199

Global Education Monitoring Report Team. (2020). Global Education Monitoring Report, 2020: Inclusion and Education: All Means All. United Nations Educational, Scientific and Cultural Organization. Recuperado de https://bit.ly/36KPxAN

Goodwin, A. L. \& Darity, K. (2019). Social justice teacher educators: What kind of knowing is needed? Journal of Education for Teaching, 45(1), 63-81. https://doi.org/10.1080/02607476.2019.1550606

González, N., Moll, L. \& Amanti, C. (2005). Funds of knowledge. Theorizing practices in households, communities, and classrooms. Taylor \& Francis Group.

Hueso, L. C. (2020). La enseñanza digital en serio y el derecho a la educación en tiempos del coronavirus. Revista de educación y derecho. Education and law review, 21(6). https://doi.org/10.1344/REYD2020.21.31283

Iglesias, E., González-Patiño, J., Lalueza, J.L., \& Esteban-Guitart, M. (2020). Por una educación crítica, intergeneracional, sostenible y comunitaria. Manifiesto en tiempos de pandemia. Revista Internacional de Educación para la Justicia Social, 9(3), 181-198. https://doi.org/10.15366/riejs2020.9.3.010 
Leiva, J. (2020). La educación intercultural e inclusiva: transformar mentalidades y contextos para el cambio y la innovación educativa. En L. Ortiz \& J. J. Carrión (Coords.) Educación inclusiva: abriendo puertas al futuro (pp. 185-201). Dykinson.

Llopart, M., \& Esteban-Guitart, M. (2018). Funds of knowledge in 21st century societies: Inclu-sive educational practices for under-represented students. A literature review. Journal of Curriculum Studies, 50(2), 145-161. https://doi.org/10.1080/00220272.2016.1247913

Lorenzo, M. M., Míguez, G. \& Cernadas, F. (2020). ¿Pueden contribuir los fondos de conocimiento a la participación de las familias gitanas en la escuela? Bases para un proyecto educativo. Teoría de la Educación. Revista Interuniversitaria, 32(1), 191211. https://doi.org/10.14201/teri.21299

Louzao, M. (2019). Educación intercultural: de la urgencia cuantitativa a la exigencia democrática. Orientaciones para la investigación y la acción en el contexto educativo asturiano. Revista de Investigación en Educación, 17(1), 44-54.

Louzao, M., Francos, M., \& Verdeja, M. (2020). Educación intercultural: algunas reflexiones y orientaciones para una práctica pedagógica en una escuela democrática y plural. Estudios pedagógicos, 46(1), 431-450. https://doi.org/10.4067/S071807052020000100431

McBride, A. E., Bellamy, D. E., \& Knoester, M. (2020). The theory and practice of developing intercultural competence with pre-service teachers on-campus and abroad. Theory Into Practice, 1-10. https://doi.org/10.1080/00405841.2020.1739957

Milner, H. R. (2011). Culturally relevant pedagogy in a diverse urban classroom. The Urban Review, 43(1), 66-89. https://doi.org/10.1007/s11256-009-0143-0

Milner, H. R. (2017). Where's the race in culturally relevant pedagogy. Teachers College Record, 119(1), 1-32.

Milner, H. R. (2015). Rac(e)ing to class: Confronting poverty and race in schools and classrooms. Harvard Education Press.

Moll, L. \& González, N. (1994). Lessons from research with language-minority children. Journal of Reading Behavior, 26(4), 439-456.

Morales, A. R. (2019). Valuing Rural Dexterity: Experiential Funds of Knowledge, Science Education, and Rural Kids. Great Plains Research, 29(1), 33-40. https://doi.org/10.1353/gpr.2019.0004

Murillo, F.J. \& Duk, C. (2020). El Covid-19 y las brechas educativas. Revista Latinoamericana de Educación Inclusiva, 14(1), 11-13. http://dx.doi.org/10.4067/S0718$\underline{737820000100011}$

Osmond-Johnson, P., Campbell, C., \& Pollock, K. (2020). Moving forward in the Covid-19 Era: Reflections for Canadian Education. EdCan Network. Recuperado de https://www.edcan.ca/articles/moving-forwardin-the-covid-19-era/

Pareja de Vicente, D., Leiva, J. \& Matas, A. (2020). Perceptions about cultural diversity and intercultural communication of future teachers. Revista Electrónica Interuniversitaria de Formación del Profesorado, 23(1), 7587. https://doi.org/10.6018/reifop.403331

Pérez-Gómez, A.I. (2012). Educarse en la era digital. Morata. 
Ríos-Aguilar, C., Kiyama, J., Gravitt \& Moll, L. (2011). Funds of Knowledge for the poor and forms of capital for the rich? A capital approach to examining Funds of Knowledge. Theory and Research in Education,9(2), 163-184.

Sanz, I., Sáinz, J., \& Capilla, A. (2020). Efeitos da crise do COVID-19 na educação. Organización de Estados Iberoamericanos para la Educación, la Ciencia y la Cultura (OEI). Recuperado de https://oei.org.br/arquivos/informe-covid-19d.pdf

Sherfinski, M., Hayes, S., Zhang, J., \& Jalalifard, M. (2020). Grappling with Funds of Knowledge in Rural Appalachia and Beyond: Shifting Contexts of Pre-Service Teachers. Action in Teacher Education, 1-22. https://doi.org/10.1080/01626620.2020.1755384

Sleeter, C. E. (2012). Confronting the marginalization of culturally responsive pedagogy. Urban Education, 47(3), 562-584.

Sleeter, C. E. (2017). Critical race theory and the whiteness of teacher education. Urban Education, 52(2), 155-169. https://doi.org/10.1177/0042085916668957

Tarabini, A. (2020). ¿Para qué sirve la escuela? Reflexiones sociológicas en tiempos de pandemia global. Revista de Sociología de la Educación-RASE, 13(2), 145155. https://doi.org/10.7203/RASE.13.2.17135

Unesco (2016). Educación 2030. Declaración de Incheon y Marco de Acción. París: Unesco.

Recuperado

de http://www.unesco.org/new/fileadmin/MULTIMEDIA/FIELD/Santiago/pdf/ESPMarco-de-Accion-E2030-aprobado.pdf

Unesco (2017). Education for sustainable development goals. Learning objectives. Education 2030. Recuperado de https://unesdoc.unesco.org/ark:/48223/pf0000247444

Unesco (2020a). Coalición Mundial para la Educación COVID-19. Recuperado de https://bit.ly/38SNMnN

Unesco (2020b). La respuesta de la Unesco al COVID-19. Recuperado de https://es.unesco.org/covid19/educationresponse.

Valenzuela, M. T. (2020). Coronavirus: un desafío global: Coronavirus: a global challenge. ARS MEDICA Revista de Ciencias Médicas, 45(1), 5-6. https://doi.org/10.11565/arsmed.v45i1.1671

Varga-Dobai, K. (2018). Remixing selfies: Arts-based explorations of funds of knowledge, meaning-making, and intercultural learning in literacy. International Journal of Multicultural Education, $20(2), \quad 117-132$. https://doi.org/10.1080/01596300903037044

Villa-Fernández, N. \& Martín-Gutiérrez, A. (2020). Educación inclusiva y digital: Desafíos y propuestas a partir del COVID-19. Revista Virtu@lmente,8(2). https://doi.org/10.21158/2357514x.v8.n2.2020.2715

Von- Braun, J., Zamagni, S., \& Sánchez-Sorondo, M. (2020). The moment to see the poor. Science, 368(6488), 214. https://doi.org/10.1126/science.abc2255 